VP-14

\title{
Laparoscopic management of perihilar cholangiocarcinoma
}

\author{
Min-Su PARK* , Bumsoo KIM, Sangmok LEE
}

Department of Surgery, Kyung Hee University, Seoul, Korea

Introduction: Surgical management of perihilar cholangiocarcinoma remains the only available treatment option with curative intent and potential influence on overall survival. Complete laparoscopic resection of perihilar cholangiocarcinoma with biliary reconstruction is a challenging procedure.

Methods: A sixty-eight-year-old man was presented with a history of fever and abdominal pain. He had a clinical and biochemical picture of obstructive jaundice with a direct bilirubin of $25 \mathrm{mg} / \mathrm{dL}$ and alkaline phosphatase of 422. His Ca 19-9 values were raised. CT scan showed an irregular wall thickening of proximal common bile duct with dilatation of upstream bile ducts (Bismuth type I). A preoperative per-cutaneous transhepatic biliary drainage was done to correct hyperbilirubinaemia.

Results: The laparoscopic resection of perihilar cholangiocarcinoma was performed with 5 trocars. The procedure included hepatoduodenal lymphadenectomy, common bile duct resection and hepaticojejunostomy. Intraoperative frozen section was performed to confirm negative resection margins. The operative time was 210 minutes with an estimated blood loss of $150 \mathrm{~mL}$. Final histopathology revealed a perihilar cholangiocarcinoma of size $1.7 \times 1.6 \times 1.3 \mathrm{~cm}$, moderately differentiated adenocarcinoma invading beyond the wall of the bile duct and nodes free of tumor (0/7), with positive lymphovascular emboli. The patient was discharged on the 9 th postoperative day with no complications.

Conclusions: The patient was discharged on the 9th postoperative day with no complications. Based on our experience, laparoscopic management of perihilar cholangiocarcinoma can be technically safe and feasible. 